\title{
Optimisation of Optical Network for Continuous-Variable Quantum Key Distribution by Means of Simulation
}

\author{
David Kobor ${ }^{1}$ and Eszter Udvary ${ }^{2}$
}

\begin{abstract}
The unprecedented breakthrough in the field of quantum computing in the last several years is threatening with the exploitation of our current communication systems. To address this issue, researchers are getting more involved in finding methods to protect these systems. Amongst other tools, quantum key distribution could be a potentially applicable way to achieve the desired level of protection. In this paper we are evaluating the physical layer of an optical system realising continuous variable quantum key distribution (CVQKD) with simulations to determine its weak points and suggest methods to improve them. We found that polarisation dependent devices are crucial for proper operation, therefore we determined their most defining parameters from the point of operation and suggested extra optical devices to largely improve transmission quality. We also paid attention to polarisation controlling in these sort of systems. Our findings could be valuable as practical considerations to construct reliable CVQKD optical transmission links.
\end{abstract}

Index Terms: quantum communication, quantum key distribution, CVQKD, optical network, simulation

\section{INTRODUCTION}

Since the first great achievements of the eighties and nineties [1], [2], quantum information technology has been drawing increasing attention, and promising groundbreaking technical solutions. However, the rapid development of quantum computing does not only have unquestionable merits, but also poses significant security threats to our existing communication networks. Thus, it is unavoidable to come up with brand new methods to ensure undisrupted operation for the future. For example, some of the most widespread encryption algorithms are relying on the very assumption, that it's rather hard - on human scale impossible - to factor large prime numbers. As quantum computers are beginning to be commissioned, this will no longer to be impossible.

A very promising field of research to protect our communication networks has been quantum key distribution (QKD), which is looking to protect the most easily exploitable part of symmetric key encryption: the distribution of common key between the communicating parties. As soon as the exchange of the secret key is considered to be secure, the proceeding communication is safe. Quantum

${ }^{1}$ BME Balatonfüred Student Research Group, Budapest University of Technology and Economics, Hungary

${ }^{2}$ Department of Broadband Infocommunications and Electromagnetic Theory, Budapest University of Technology and Economics, Budapest, Hungary

1,2E-mail: david.kobor@edu.bme.hu; udvary@hvt.bme.hu key distribution can be divided to three major categories: discrete-variable QKD, continuous-variable QKD and distributed-phase-reference QKD. In this paper the focus is on continuous-variable QKD (CVQKD). CVQKD offers the major advantage of not requiring any special, high-cost components, but might be built up using only conventional telecommunication devices [3]. This fact makes it relatively easy and straightforward to implement and measure test devices. To ensure the highest possible key rate all noise contributions of the link must be kept as low, as possible, regardless of the external or internal source. This the reason why CVQKD connections are in many cases realised over optical fibre, but there have also been efforts to establish a connection over free-space [4] and evaluating free space transmission [5].

In 2008 the European Integrated Project (SECOQC) team proposed a working CVQKD connection over $8 \mathrm{~km}$ of optical fibre, at $8 \mathrm{kbps}$ key rate [6], [7]. The aim of Symmetric Encryption with QUantum key REnewal (SEQURE) project has been the same. They maintained quantum secured communication over $12 \mathrm{~km}$ of fiber at a maximum of 1 kbps key rate. The Budapest University of Technology and Economics (BME) have also started developing a setup for quantum key distribution to demonstrate its feasibility [8]. In the last couple of years great effort has been devoted to the difficulties of practical implementation. Researchers are looking for methods of extending the link range (e.g. with new protocols [9]), maintaining connection over different mediums [10], [11], and trying to optimise electrical or optical components of the complex system [12], [13], as well as giving better theoretical description of the employed devices [14]. In this paper we are taking a different approach and use classical optical system simulation (VPI Transmission Maker) to evaluate the optical layer of a CVQKD network in order to optimise the parameter choice, and come up with suggestions regarding the specific optical devices. Our goal is to conduct simulations prior to building the quantum link, to get an idea, what level of transmission quality might be expected from the system. CVQKD has been investigated from many points of view, the theoretical basis of this system has already been worked out in [8], but there has been very little discussion about the actual physical construction of such systems (for example how to choose the optical components and what to look after when assembling them). We are suggesting minor changes in the already proposed 
architecture, focusing on weak points in the design of the physical layer to improve on it to the highest extent possible. Our design considerations might not only be utilised for system comprising of discrete components, but could also be useful for integrated photonic chip design for CVQKD [15].

In Sec. 2 we are describing the operation of the system on the level of the optical network, list several important consideration that we kept in mind during the simulations. In Sec. 3 we detail the most important undesired mechanisms we identified that are affecting system performance. In Sec. 4 we give an overview of our polarisation controlling method and its impairments. Sec. 5 is to summarise our findings and draw the conclusions.

\section{System Simulation}

In this section we describe the network we had implemented in the simulation environment, as well as the most important consideration we kept in mind before and during the simulations.

\section{A. Model}

The block diagram of the system is depicted in Fig. 1. Extremely low energy impulses are used to communicate between the two parties (Alice and Bob), whose behaviour might be described using the laws of quantum physics. Basically, it is operating as a self-seeded homodyne optical transmission system with balanced detector structure, however certain modifications are applied to ensure flawless quantum operation. The transmitter is producing complex numbers coded in the quadrature of extra-low energy light impulses. Quantum operation is based on the sufficiently low energy of these pulses.

The optical carrier is produced on Alice's side by a laser, which is protected with an isolator, while the CW light is modulated with high extinction intensity modulator to get light impulses of certain periodicity. In ideal operation the laser light is assumed to be perfectly linearly polarised. In the figure this is marked with the red signal. The impulses are split in 90:10 ratio in two separate paths: the reference for homodyne detection and the signal carrying the actual information (modulated signal). $90 \%$ of power is going directly to the output of the transmitter (PBS 2), while the $10 \%$ is intensity and amplitude modulated and attenuated even further to reach the desired power level. The cumulative attenuation of the modulated path should be set in such way, that the transmitted packets contain at most a few hundred photons. This low transmitted power per packet ensures the overall security of the system. The path of the modulated signal is extended with an additional structure seen in Fig. 1. This signal is routed to the $\mathrm{X}$ polarised port of a polarisation beam-splitter (PBS 1), where $X$ is the assumed polarisation state of the laser. This routes the signal to the common port of the PBS (XY of PBS 1), where it is passed through an optical delay line and is reflected by a Faraday-mirror (FM). The FM rotates the polarisation of the signal by $90^{\circ}$ and reflects it back to PBS 1 through the delay line again, where it is now exiting the other ( $\mathrm{Y}$ polarised) port. Here it gets recombined with the reference signal, but now the impulses are not only separated in time, but are also orthogonal in polarisation (due to the effect of FM). These optical functions (delay and polarisation rotation) are applied to ensure minimal interference between the modulated and the reference signal while propagating on the long optical fibre connecting Alice and Bob.

During the propagation on the fibre the state of polarisation of the signal gets further rotated by an unpredictable amount. To overcome this we use a polarisation controller to reset the initial state we had on the transmitter output. This is a crucial point, because in the next we seek to separate the reference and the signal again. We use a polarisation beam splitter (PBS 3) for this function and successful separation requires perfectly set polarisation. The modulated signal gets directly to the balanced receiver. On the reference path the reference signal is routed to the very same structure what we had in the transmitter (PBS 4). This time the reference signal is rotated in polarisation and delayed, to be in the same time and polarisation state as the modulated signal, when it reaches the balanced receiver.

\section{B. Simulation Considerations}

- We are using VPI Transmission Maker to build the model of the system depicted above. This software has been developed to evaluate conventional optical transmission links based on classical physical principles, therefore it is unable to take any quantum behaviour into consideration. However, we are not looking to demonstrate any complex key-sharing operation or any protocol level use. Our goal is to improve the optical layer of the key sharing system, by achieving the lowest noise and self-interference, which might be successfully done with classical methods.

- The architecture described above is the very same, that has been built at the Budapest University of Technology and Economics. We were using the parameters of the devices they employed based on their data sheets.

- The laser source is not perfectly linearly polarised. We didn't find any information about the exact grade of imperfection in the data sheet, but therefore we assumed a power ratio of 100 (20 dB polarisation extinction ratio) between the orthogonal polarisation axes.

- Polarisation management is utterly important throughout the whole system, however most of the data sheets don't detail polarisation dependent operation. Conventional beam splitters and isolators are not polarisation maintaining (PM) elements. There are available PM devices, but these also affect the polarisation, however their effect is much smaller than that of the conventional components. This contribution might be expressed with an angle of rotation. In case of all PM and non-PM devices we approximated a degree of rotation on the polarisation states to describe their behaviour. We are also assuming a worst-case situation, meaning that all rotations are performed in the same direction, the effect of the succeeding devices don't cancel out. 


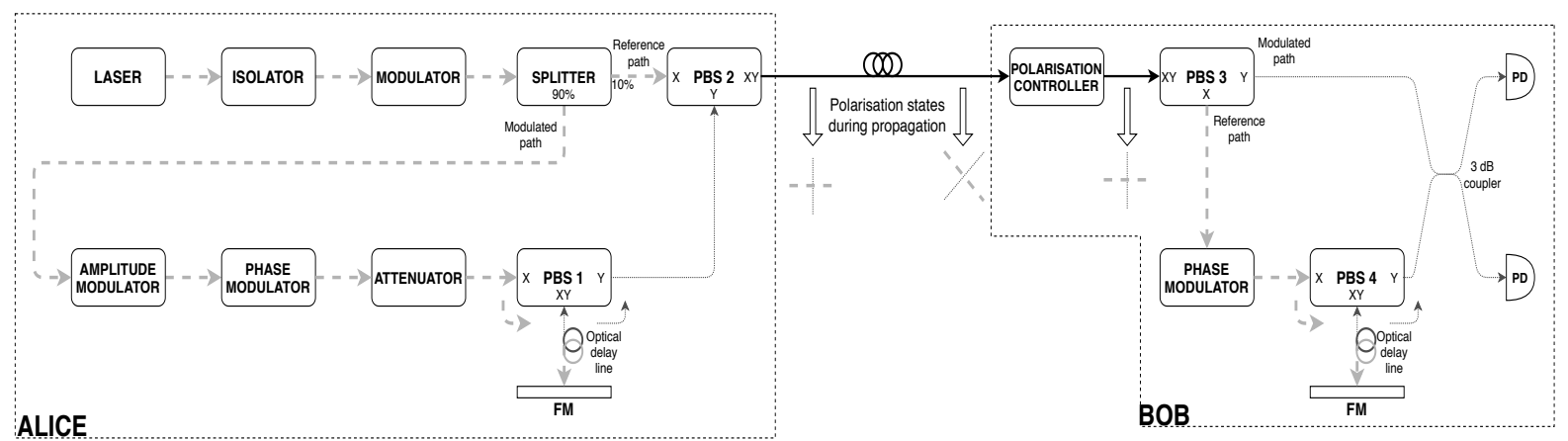

Fig. 1. Block diagram of CVQKD optical system; two orthogonal polarisation states marked with light grey dashed line (X) and dark grey dotted line (Y); the transmitter (Alice) produces the pulsed optical carrier, which is split in two paths of which one is modulated and has its polarisation rotated. The two paths are then combined and sent to the receiver (Bob), where the signal is split again based on polarisation and finally detected with a coherent receiver. PBS 4 is used to match the polarisation of signals in the two branches.

- Polarisation beam splitters (PBS) and combiners are employed for two purposes in our system. First we use them to separate and join the reference signal and the one carrying the actual information. This happens at the output of the transmitter and the input of the receiver. We also use PBSs for a different purpose: to construct circulator-like sub-systems to delay and change the state of polarisation of the signals. We are going to use the graphical representation of the PBS seen on Fig. 1 (PBS 1) for further explanation. In case of our circulatorlike structures the light enters at one of the polarised ports (X) and exists the common port (XY). When it is reflected by the Faraday-mirror, it goes through $\mathrm{XY}$ again, but this time exits the device at the other polarised port (Y), because its polarisation has been modified. The manufacturer defines the polarisation extinction ratio (PER): when perfectly linearly $\mathrm{X}$ polarised light enters the common port (XY), its full power should be exiting the dedicated port $(\mathrm{X})$. Due to the imperfections some $\mathrm{X}$ polarised light gets to the Y port, and PER tells us how significant this contribution is. This is crucial parameter in our application, and it must be modelled very carefully. There is another important parameter, which we could not find in any data sheet: the crosstalk between the singular ( $\mathrm{X}$ and $\mathrm{Y}$ ) ports. As we feed an optical signal to the $\mathrm{X}$ port, its full power is bound to exit on the common port (XY). But based on our experiences, there is a tiny fraction of light, that gets immediately reflected from the $\mathrm{XY}$ port and goes directly to $\mathrm{Y}$ by skipping the optical delay line and Faraday-mirror. According to our measurements this crosstalk should be in the domain of $-60 \mathrm{~dB}$, which is rather low, but still crucial in our CVQKD optical system. This had to be modelled very carefully.

- We didn't have any information regarding the polarisation dependent behaviour of phase and amplitude modulators, therefore we assumed ideal operation.

\section{Evaluation}

We are treating the CVQKD optical system as a conventional transmission system, therefore we utilise classical methods of evaluation. For this purpose we utilised simple QAM and PSK modulation schemes and we took their error vector magnitude (EVM) as a descriptive metrics. Moreover, we have also taken the time domain waveforms produced by the simulator, so we can learn more about the nature of flaws and weaknesses during the operation.

\section{Simulation Results}

1000 randomly generated symbols have been sent during the simulations, while we were looking at the EVM and waveforms. The definition of EVM might be seen below:

$$
\operatorname{EVM}(\%)=\sqrt{\frac{P_{\text {error }}}{P_{\text {reference }}}} \times 100 \%
$$

The symbols of reference for are known, the error vectors can easily be calculated from the simulation results. We have identified two important mechanisms that have a significant impact on transmission quality and need to be addressed. We are using this section to describe these mechanisms.

\section{A. Insufficient separation of reference and modulated signals}

The complete system seen on Fig. 1 is constructed to provide the best possible separation between the reference signal (LO) and the modulated signal and minimise crosstalk. This is the reason for using Faraday-mirror and optical delay line. However, mainly due to the imperfections of the employed optical devices this is not always enough. Considering that the reference signal has a much higher power than the modulated signal, it is able to completely ruin the operation. Even if we use devices sold as polarisation maintaining, we can be sure that they are not performing perfectly, they have a certain degree of polarisation changing effect. The same goes for the laser, whose cross-polarisation suppression can never be infinite. The polarisation controller is only able to compensate the impairments caused by the fibre connecting Alice and Bob. This is why we have to be very considerate in the design phase when choosing the device parameters. Our splitter (seen in Fig. 1) is splitting the optical carrier in 90:10 ratio, where the $90 \%$ is the reference signal. At this point we assume, that the light is linearly polarised, but this is not true. In fact, after the splitting the optical carrier is going to be mainly polarised 


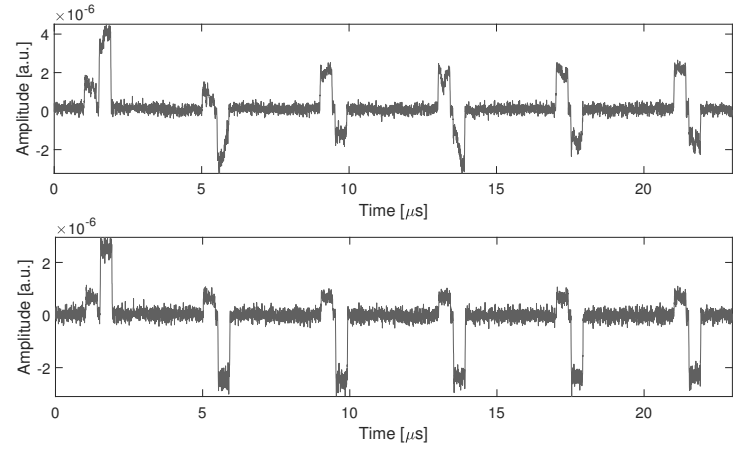

Fig. 2. Comparison of output waveforms without and with polariser on Alice's side

along the $\mathrm{X}$ axis on both routes, but a certain fraction is going to be orthogonal to it $(\mathrm{Y})$. On the reference route this light is going to the output polarisation combiner (PBS2), while its $\mathrm{X}$ aligned component is desired, its $\mathrm{Y}$ aligned component is undesired. On the modulation route the $10 \%$ of power is further attenuated and transformed to be Y polarised (using the Faraday-mirror). Subsequently the modulated signal is routed to PBS 2 and recombined with the reference signal. The issue in that the modulated signal is $\mathrm{Y}$ polarised on purpose, while the $\mathrm{X}$ polarised reference signal also has a fraction of $\mathrm{Y}$ polarised power. These Y polarised contributions have about the same magnitude of power, because the modulated signal has been strongly attenuated, while the reference signal hasn't been. They propagate over the same medium connecting Alice and Bob, thus we must expect considerable interference. We have found and simulated several methods to avoid this.

- The first idea could be to insert a polariser after the laser source to improve its linearly polarised behaviour. According to our simulation this is not a desired method, because all subsequent devices also modify the polarisation, therefore when the light reaches the critical place (PBS 2), it is not going to be well polarised anymore.

- We have found the place in the system to use a polariser with the highest efficiency in minimising the interference of the modulated and reference signals. It is to be used between the Splitter and PBS 2 in the reference route, in order to decrease the power propagating in $\mathrm{Y}$ polarisation state on the reference route. This minimises the power interfering with the modulated signal in the Y (marked with blue) polarisation state. Fig. 2 gives an idea about the difference that this polariser makes. The upper plot of Fig. 2 is depicting the output waveform without polariser, while the lower one with it. It can be seen that the well placed polariser make the impulses much more symmetric, more square wave-like. The improvement is significant.

- We might achieve the same grade of polarisation of the reference signal with less explicit modification. If a PBS 2 is chosen to have high polarisation extinction ratio (PER), it will act better at suppressing the Y polarised contribution of the reference signal before routing to the common
(XY) port. However, a commercially available polariser works with at least $20 \mathrm{~dB}$ of additional suppression in the undesired polarisation state, while increasing the PER of a normal PBS by $20 \mathrm{~dB}$ is rather unrealistic.

To conclude the above, the best method to further increase the separation of the modulated and reference signals is to make the reference signal more polarised before recombining it with the modulated one. The easiest and most straightforward way to this is to extend the setup with a $\mathrm{X}$ aligned polariser between the Splitter and PBS 2.

\section{B. Pre-impulses}

After the first simulations we have noticed unexpected, small amplitude impulses before every normal (expected) impulse on the receiver side (also shown in Fig. 2). They appeared $500 \mathrm{~ns}$ before the expected impulses, which was the exact amount of delay caused by the optical delay line in the circulator-like structure. This allowed us to conclude, that these pre-impulses impulses are present, because a certain fraction of optical power bypasses this delay line, meaning that there is no full separation between the polarised individual ports ( $\mathrm{X}$ and $\mathrm{Y}$ polarised) of the polarisation beam splitters. The principle of the operation requires both reference and modulated signal in the receiver to actually produce an output signal, otherwise we wouldn't get pre-impulses.

At first glance it would seem that the root of this impairment is a tiny fraction of modulated optical signal avoiding PBS 1 on Alice side and finding its way to the balanced receiver, while a small fraction of the reference signal is also bypassing PBS 4 in Bob's device. In this scenario pre-impulses are forming the same way as the useful, high amplitude impulses, but with bypassing the circulator-like structures on both sides. In this case we should be seeing a small power copy before all impulse, but this is not what we experienced. Our simulations showed that pre-impulses are always having the same polarity (regardless of the polarity of the subsequent useful impulse), and are only measured in one quadrature. With further simulations we have proven that PBS 1 has no effect whatsoever on the pre-impulses. Thus, the original assumption is false, the answer must be found on Bob's device.

The actual mechanism causing this issue is only the reference signal itself. The reference signal is exiting Alice's receiver with small attenuation in its way, consequently it will enter Bob's receiver with a relatively large power. After the polarisation controller it is routed to the phase modulator by PBS 3, but a small amount of $\mathrm{X}$ polarised reference signal will immediately get to the balanced receiver through the $\mathrm{Y}$ port of PBS 3 with no delay (due to imperfections of polarisation splitter defined by PER). At the same time most of its power passes the phase modulator and is routed to PBS 4, where in theory it should be delayed and have its polarisation modified.

Because PBS 4 also has a certain grade of crosstalk (about $60 \mathrm{~dB}$ ) between its $\mathrm{X}$ and $\mathrm{Y}$ ports, a small fraction of power is not delayed, but is going immediately in the balanced receiver. The proof of this concept is that the magnitude of pre-impulses 
TABLE I

EFfect of Parameter Modification of Certain Devices Expressed in EVM

\begin{tabular}{|c|c|c|c|}
\cline { 2 - 4 } \multicolumn{1}{c|}{} & Modification & EVM & Change in EVM \\
\hline 1 & N/A (reference) & $4.39 \%$ & $0 \%$ \\
\hline 2 & Alice polariser 15 dB $\Rightarrow 25 \mathrm{~dB}$ & $3.30 \%$ & $1.09 \%$ \\
\hline 3 & Alice polariser 15 dB $\Rightarrow 35 \mathrm{~dB}$ & $3.17 \%$ & $1.22 \%$ \\
\hline 4 & Alice PBS 1 PER 20 dB $\Rightarrow 30 \mathrm{~dB}$ & $4.35 \%$ & $0.04 \%$ \\
\hline 5 & Alice PBS 1 PER 20 dB $\Rightarrow 40 \mathrm{~dB}$ & $4.34 \%$ & $0.05 \%$ \\
\hline 6 & Alice PBS 2 PER 20 dB $\Rightarrow 30 \mathrm{~dB}$ & $3.30 \%$ & $1.09 \%$ \\
\hline 7 & Alice PBS 2 PER 20 dB $\Rightarrow 40 \mathrm{~dB}$ & $3.16 \%$ & $1.23 \%$ \\
\hline
\end{tabular}
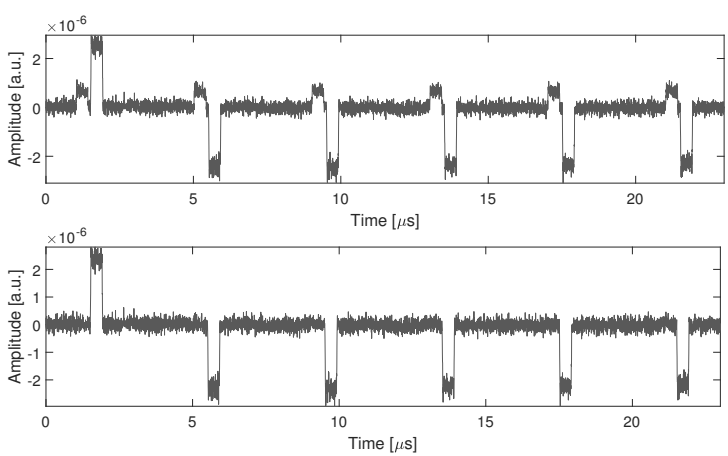

Fig. 3. Comparison of output waveforms without and with polariser on Bob's side

is independent of all devices on Alice's side, however the polarisation extinction ratios of the PBSs in Bob's device affect it: choosing a larger PER reduced the pre-impulse magnitude. Suggestions to address this problem:

- First we should choose PBSs for our setup, those have a larger grade of separation between their polarised ports ( $\mathrm{X}$ and $\mathrm{Y}$ ). This is rather hard to do, because manufacturers don't usually optimise this specific parameter, this is not even included in most of the PBS data sheets.

- We might also look for PBSs with larger PER. This is one of the most important parameters to look for in the data sheet, much more realistic, than optimising the crosstalk between $\mathrm{X}$ and $\mathrm{Y}$ ports.

- In case we don't have any further chance to swap the existing the PBSs on Bob' side, employing a polariser can also make a huge difference. The main cause of the problem (pre-impulses) is the $\mathrm{X}$ polarised reference signal routed in the same place with the $\mathrm{Y}$ polarised modulated signal, namely to the $\mathrm{Y}$ port of PBS 3 . We might reduce this $X$ polarised undesired power by using a $\mathrm{Y}$ aligned polariser between the PBS 3 and the balanced receiver. We are showing the effect of this polariser in Fig. 3. By comparing the two plots it might be seen that the pre-impulses ceased to exist on the lower one due to the polariser.

\section{Numerical results}

In Table 1 we summarise the most important parameters of the polarisation dependent components affecting the transmission quality. We express the results in EVM. The first line is giving the EVM in the base parameter setting (reference), the other lines all contain one modification compared to this state. These changes basically all have the same physical effect, they make the light more polarised in their location. The most explicit way to do this is the use of polariser as seen in the second and third row of Table 1. By increasing the polarisation extinction ratio of the output combiner on Alice's side, we get the same result. This device polarises both the modulated and reference signals, therefore it is a bit more effective than the polariser, which only affects the reference signal. This becomes apparent if we compare row 3 with row 7. However, increasing the PER beyond a certain level is basically impossible due to manufacturing difficulties, so it is recommended to use a polariser to experience about the same result, at lower cost and effort. By improving the parameters of the PBS in the circulator like structure in the transmitter (PBS 1), less significant changes can be observed. This is because PBS 1 only deals with the modulated signal, while from separation point of view the more important one to handle is the reference signal. According to our simulations, all the other passive polarisation dependent components didn't have considerable effect on transmission quality.

\section{POLARISATION CONTROLLING}

Polarisation controlling has dedicated importance for proper operation. In our CVQKD system we are using a polarisation controller to correct the polarisation changing effect of the standard fibre connecting Alice and Bob. If it's not working properly, PBS 3 will not be able to correctly separate the modulated and reference signals resulting in an error. The CVQKD system at our university utilises a General Photonics POS-002 controller. It is working to maximise the power measured of a reference point chosen by us. The location of the controller is fixed (Bob's input), but we are free to choose the reference point. In this section we are dealing with the proper choice for reference point, moreover we are evaluating the effect of controlling error.

\section{A. Reference point}

The root cause of most of the previously detailed impairments is the interference of the modulated and reference signals, mainly because of the unsatisfying behaviour of polarisation splitters and combiners (these two are physically identical). The polarisation controller is basically doing the same job, it is trying to minimise interference, therefore we must choose the location of the reference very carefully 


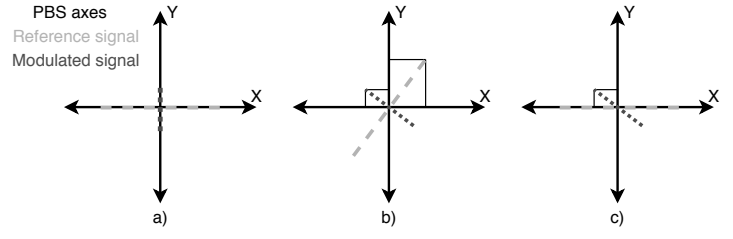

Fig. 4. Schematic operation of polarisation splitters

to address this issue. We also know - based on previously detailed considerations -, that the leakage of the reference signal into the routes of the modulated signal poses with the biggest threat. Thus the modulated and reference signals are not of equal importance, we have to pay more attention to the proper separation of the reference optical signal.

Fig. 4 is a simple depiction of how the polarisation splitter sets its $\mathrm{X}$ and $\mathrm{Y}$ (slow and fast axis in other words) outputs. Fig. 4.a depicts an ideal scenario, in which the reference signal (marked with red) is linearly polarised and its full power is matched with the $\mathrm{X}$ axis of the PBS. The same goes for the modulated signal (marked with blue), its power is in the orthogonal state of polarisation and is perfectly aligned with the $\mathrm{Y}$ axis. In this case the PBS is able to separate the two, leaving no interference: $\mathrm{X}$ output will only have the reference signal, Y output only the modulated one. In case Fig. 4.b the two signals are still orthogonal in polarisation, but there is a slight rotation compared to the axes of the PBS. This is the case of a certain degree of polarisation controlling error. The PBS is unable to separate the two, they are going to interfere. $\mathrm{X}$ and $\mathrm{Y}$ outputs will contain some of both the modulated and some of the reference signal, depending on the projection, as seen in Fig. 4. It is apparent, that we are looking to achieve scenario Fig. 4.a. We also must see, that the polarisation of the two signals will not always be orthogonal. Its because that they are processed in separate optical paths, travelling through different components with various properties, so they will not be orthogonal when recombined again. This is shown on Fig. 4.c. In case Fig. 4.b, if we use good polarisation controlling mechanisms, we are able to perfectly split the two signals, in case Fig. 4.c this will be impossible. This must be kept in mind when choosing the reference point for the controller, because Fig. 4.c is exactly what is happening in our system. We must be careful to keep concentrate the reference optical power to one output not to interfere with the modulated signal. Power leakage in the other way is accepted, due to the large differences in power. To draw the conclusion, the reference point must be at the $\mathrm{X}$ output of PBS 3 , to concentrate the reference power in that path.

\section{B. Controlling error}

In the Table 2 we are giving an overview of the effect of different magnitudes of polarisation controlling errors. We are starting from 0.001 degrees and moving with logarithmic steps to $1^{\circ}$. Controlling error will affect the efficiency of PBS 3 in separating the reference and modulated signals. This error
TABLE II

EFFECT OF ERROR IN POLARISATION CONTROLLING

\begin{tabular}{|c|c|c|}
\hline Error & EVM (\%) & Difference (\%) \\
\hline $0^{\circ}$ & 3.4654 (reference) & 0 \\
\hline $0.001^{\circ}$ & 3.4656 & 0.0002 \\
\hline $0.01^{\circ}$ & 3.4930 & 0.0276 \\
\hline $0.1^{\circ}$ & 4.9620 & 1.4966 \\
\hline $1^{\circ}$ & 33.1339 & 29.6685 \\
\hline
\end{tabular}

cannot be corrected later in the system, therefore it is crucial achieving the best possible operation.

As seen on Table $1,0.01^{\circ}$ error is not a significant error, but with $0.1^{\circ}$ the EVM is starting to rise dramatically. At $1^{\circ}$ the transmission is basically collapsed. $0.1^{\circ}$ error might be expressed in $\mathrm{dBs}$ : it resembles $-55 \mathrm{~dB}$ of crosstalk between the reference and modulated signals. It might seem to be low, but as mentioned earlier, the system is very sensitive due to the large power differences.

\section{CONCLUSION}

In this paper we used simulations to evaluate the performance of an optical transmission network suitable for continuous-variable quantum key distribution (CVQKD) to improve its performance. While most papers on this field focus on the issue of quantum theory, protocols and the construction of novel architectures, we gave practical considerations for the construction of a specific systems from a photonic engineer's point of view. We have proven that the crucial passive components in the system are the polarisation dependent devices, mainly the polarisation splitters and combiners (PBSs), whose insufficient behaviour reduce the separation of the modulated and reference signal impulses. We have shown that the proper parameter choice of PBSs is very important, and the transmission quality may be further improved using polariser. We have found the best possible location for extra polarisers in the system and validated our assumption in theory and also with simulations. This turned out to be a very straightforward modification, because a relatively simple and cheap passive device in the right place makes a large difference in terms of transmission quality. We also looked at the question of polarisation control, which is a defining factor for long-term stability. We simulated what magnitude of error might be accepted in the system and also found the ideal operation conditions for our specific controller. Our results might be able to give guidelines for the construction of CVQKD systems on the level of the optical backhaul network.

\section{ACKNOWLEDGMENT}

The research has been supported by the European Union, co-financed by the European Social Fund (EFOP-3.6.2-162017-00013, Thematic Fundamental Research Collaborations Grounding Innovation in Informatics and Infocommunications). The authors would like to special thank Dr. Zsolt Kis for the helpful suggestions. 
Optimisation of Optical Network for Continuous-Variable

Quantum Key Distribution by Means of Simulation

\section{REFERENCES}

[1] P. W. Shor. Algorithms for quantum computation: discrete logarithms and factoring. Proceedings 35th Annual Symposium on Foundations of Computer Science. Santa Fe, NM, USA, 1994, pp. 124-134. Dor: 10.1109/SFCS.1994.365700

[2] Bennett, Charles \& Zekrifa, Djabeur Mohamed Seifeddine. Quantum cryptography: Public key distribution and coin tossing. Theoretical Computer Science - TCS, 1984, vol. 560, p. 175-179.

[3] Marie, Adrien \& Alleaume, Romain.. Self-coherent phase reference sharing for continuous-variable quantum key distribution. Physical Review, 2016, A. 95. Dor: 10.1103/PhysRevA.95.012316.

[4] Bisztray, Tamas \& Bacsardi, Laszlo. (2018). The evolution of freespace quantum key distribution. Infocommunications Journal. 10. 22-30.

[5] Galambos, M. \& Bacsardi, Laszlo. (2018). Comparing calculated and measured losses in a satellite-Earth quantum channel. Infocommunications Journal. 10. 14-19.

[6] Poppe, Andreas \& Peev, Momtchil \& Maurhart, Oliver. Outline of the SECOQC quantum-key-distribution network in Vienna. International Journal of Quantum Information, 2008, 6. 209-218. DoI: $10.1142 / \mathrm{S} 0219749908003529$.

[7] Peev, Momtchil et al., The SECOQC quantum key distribution network in Vienna. New Journal of Physics, 2009, 11. 075001. DoI: 10.1088/1367-2630/11/7/075001.

[8] Mraz, Albert et al., (2017). Quantum circuit-based modeling of continuous-variable quantum key distribution system: SIMULATION RESULTS OF A NOVEL CVQKD CIRCUIT. International Journal of Circuit Theory and Applications. Dor: 10.1002/cta.2347.

[9] P. Jouguet, S. Kunz-Jacques, A. Leverrier, P. Grangier And E. DIiamanti. Experimental demonstration of continuous-variable quantum key distribution over $80 \mathrm{~km}$ of standard telecom fiber. CLEO: 2013, San Jose, CA, 2013, pp. 1-2.

[10] N. Hosseinidehaj, Z. Babar, R. Malaney, S. X. Ng AND L. Hanzo. Satellite-Based Continuous-Variable Quantum Communications: Stateof-the-Art and a Predictive Outlook. IEEE Communications Surveys \& Tutorials, vol. 21, no. 1, pp. 881-919, Firstquarter 2019. DoI: 10.1109/COMST.2018.2864557

[11] Z. Qu And I. B. Djordjevic. RF-assisted coherent detection based continuous variable $(\mathrm{CV}) \mathrm{QKD}$ with high secure key rates over atmospheric turbulence channels. 2017 19th International Conference on Transparent Optical Networks (ICTON), Girona, 2017, pp. 1-5. DoI: 10.1109/ICTON.2017.8024919

[12] Xiaoxiong, Zhang \& Zhang G, Yi-Chen \& Li, Zhengyu \& Yu, Song \& Guo, Hong. 1.2 GHz Balanced Homodyne Detector for ContinuousVariable Quantum Information Technology. IEEE Photonics Journal, 2018, PP. 1-1. DoI: 10.1109/JPHOT.2018.2866514.

[13] X. Wang, J. Liu, X. Li And Y. Li. Generation of Stable and High Extinction Ratio Light Pulses for Continuous Variable Quantum Key Distribution. IEEE Journal of Quantum Electronics, June 2015, vol. 51, no. 6, pp. 1-6, Art no. 5200206. Dor: 10.1109/JQE.2015.2427031

[14] P. Kucera, Quantum Description of Optical Devices Used in Interferometry. Radioengineering, 2007, vol. 16, no. 3.

[15] Sibson, Philip \& Kennard, Jake \& Stanisic, Stasja \& Erven, Chris \& O'Brien, Jeremy \& G Thompson, Mark. ntegrated Silicon Photonics for High-Speed Quantum Key Distribution. Optica, 2016, 4. DoI: $10.1364 /$ OPTICA.4.000172.

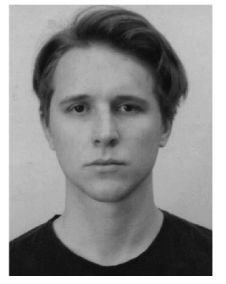

David Kobor received his B.Sc. from the Budapest University of Technology and Economics (BME), in 2018. He is a member of the Balatonfüred Student Research Group. He is currently pursuing a Master's degree at the same institute specialised in Wireless Networks and Applications. His professional interests include simulation and measurement of optical systems, such as Radio over fibre applications and optoelectronic oscillators.

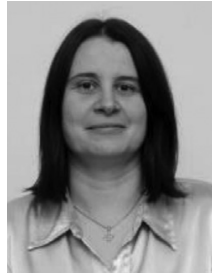

Eszter Udvary received a $\mathrm{Ph} . \mathrm{D}$. degree in electrical engineering from Budapest University of Technology and Economics (BME), Budapest, Hungary, in 2009. She is currently an Associate Professor at BME, Department of Broadband Infocommunications and Electromagnetic Theory, where she leads the Optical and Microwave Telecommunication Lab. Dr. Udvarys research interests are in the broad areas of optical communications, include optical and microwave communication systems, Radio over fibre systems, optical and microwave interactions and applications of special electro-optical devices. 